Research Paper

\title{
Albumin and Fibrinogen Combined Prognostic Grade Predicts Prognosis of Patients with Prostate Cancer
}

\author{
Yanqing Wang1*, Wei Chen ${ }^{2 *}$, Chuanyi Hu${ }^{3 *}$, Xiaofei Wen ${ }^{4 *}$, Jiahua Pan ${ }^{1}$, Fan $\mathrm{Xu}^{1}$, Yinjie Zhu1 ${ }^{1}$, Xiaoguang \\ Shao ${ }^{1}$, Xun Shangguan ${ }^{1}$, Liancheng Fan¹, Jianjun Sha ${ }^{1}$, Zezhou Wang5 ${ }^{5}$, Yong Cai ${ }^{5}$, Qiang Liu 6 , Baijun \\ Dong $^{1 凶}$, Wei Xue ${ }^{1 \bowtie}$ \\ 1. Department of Urology, Renji Hospital, School of Medicine, Shanghai Jiao Tong University, Shanghai, China; \\ 2. Department of Urology, Zhongshan Hospital of Fudan University, Shanghai, China; \\ 3. Department of Urology, Shanghai Pudong New Area Gongli Hospital, Shanghai, China; \\ 4. Department of Urology, East Hospital, Tongji University School of Medicine, Shanghai, China; \\ 5. School of Public Health, Shanghai Jiao Tong University, Shanghai, China; \\ 6. Department of Pathology, Renji Hospital, School of Medicine, Shanghai Jiao Tong University, Shanghai, China. \\ * The first four authors contributed equally to the manuscript. \\ $\triangle$ Corresponding authors: Baijun Dong, Department of Urology, Renji Hospital, School of Medicine, Shanghai Jiao Tong University, 1630 Dongfang Road, \\ Pudong District, Shanghai, 200127, P. R. China. (Phone: (86) 21-6838-3757, Fax: (86) 21-5870-9251, E-mail: dongbaijun@renji.com). Wei Xue, Department of \\ Urology, Renji Hospital, School of Medicine, Shanghai Jiao Tong University, 1630 Dongfang Road, Pudong District, Shanghai, 200127, P. R. China. (Phone: (86) \\ 21-6838-3716, Fax: (86) 21-5870-9251, E-mail: xuewei@renji.com). \\ (c) Ivyspring International Publisher. This is an open access article distributed under the terms of the Creative Commons Attribution (CC BY-NC) license \\ (https://creativecommons.org/licenses/by-nc/4.0/). See http://ivyspring.com/terms for full terms and conditions.
}

Received: 2017.05.17; Accepted: 2017.08.31; Published: 2017.10.23

\begin{abstract}
Background: The nutritional status and systemic inflammation are thought to be associated with outcome in multiple types of cancer. The objective of this study was to determine the prognostic value of pretreatment albumin and fibrinogen combined prognostic grade (AFPG) in prostate cancer (PCa).

Methods: 462 prostate cancer patients who had undergone androgen deprivation therapy (ADT) as first-line therapy at four cencters were retrospectively analyzed. The serum albumin levels and plasma fibrinogen levels were measured at the time of diagnosis. The AFPG was calculated according to albumin and fibrinogen levels dichotomized by optimal cut-off values or clinical reference values. Univariate and multivariate cox regression analyses were performed to determine the associations of AFPG with progression-free survival (PFS), cancer-specific survival (CSS) and overall survival (OS). Prognostic accuracy was evaluated with the Harrell concordance index.

Results: Multivariate analyses identified AFPG as an independent prognostic indicator for PFS, CSS and OS (each $p<0.01$ ). According to optimal cut-off values, the addition of AFPG to the final models improved predictive accuracy for PFS, CSS and OS compared with the clinicopathological base models, which included Gleason score and incidence of metastasis. Moreover, AFPG according to optimal cut-off values was a better prognostic predictor than albumin levels alone or fibrinogen levels alone or AFPG according to clinical reference values.

Conclusion: Decreased AFPG could predict a significantly poor prognosis in patients with PCa. Thus, we recommend adding AFPG according to optimal cut-off values to traditional prognostic model to improve the predictive accuracy.
\end{abstract}

Key words: prostate cancer; albumin; fibrinogen; prognosis; biomarker.

\section{Introduction}

The morbidity and mortality of prostate cancer $(\mathrm{PCa})$ in China is increasing in recent years, it is estimated that there will be about 60,300 newly diagnosed PCa and 26,600 Chinese will die from PCa in 2015, and the proportion of aged or advanced or metastatic PCa patients in all PCa patients is higher than that in western countries [1]. Androgen deprivation therapy (ADT) is the mainstay of therapy for patients with locally advanced or metastatic PCa or patients with early-stage disease who are ineligible 
for local regional treatments due to health disparity [2].

A great deal of evidence indicates that the prognosis of patients with cancer is associated with the nutritional status and systemic inflammatory response to tumor [3-5]. Serum albumin level, which is commonly used to assess the nutritional status, is an important prognostic factor in advanced cancer [6]. Sejima $\mathrm{T}$ et al. [7] reported that low pre-operative levels of serum albumin could predict lymph node metastases and ultimately correlated with a biochemical recurrence of prostate cancer in radical prostatectomy patients. A number of systemic inflammation-based parameters including C-reactive protein (CRP) [5], neutrophil to lymphocyte ratio (NLR) [8], platelet to lymphocyte ratio (PLR) [9], have been reported to be associated with the prognosis of PCa. Nutrition and inflammation are not independent, but regulated with each other [10]. Based on this, a novel inflammation-based prognostic system named the combination of systemic inflammation and nutritional status was increasingly investigated, such as modified Glasgow prognostic score which defined as the combination of CRP and albumin [11], albumin and neutrophil combined prognostic grade which defined as the combination of albumin and neutrophil [12], Inflammatory-Nutritional Index which defined as ratio of albumin to CRP [13]. However, CRP is not commonly used in clinic for its low sensitivity and unconventional detection. Similar to CRP, the plasma fibrinogen is also an acute-phase reaction protein of host response to tumor [10], but its measurement is routinely performed. Besides, we have showed an association beween elevated plasma fibrinogen with poor clinical outcome for PCa patients [14].

Based on aboved studies, we proposed a new index named albumin and fibrinogen combined prognostic grade (AFPG), and hypothesized that pretreatment AFPG was a valuable prognostic indicator for PCa. The objective of this study was to verify our hypotheses and evaluate whether it could improve the predictive accuracy for prognosis of PCa.

\section{Materials and Methods}

\section{Study population}

After obtaining approval from the Ethics Committee at four hospitals, and informed consent from patients, medical records of 535 PCa patients who had undergone ADT as first-line therapy at four centers between January 2010 and December 2014 were retrospectively reviewed. We excluded patients with hepatopathy, coagulation-related diseases, inflammatory diseases, autoimmune diseases, cardiovascular and cerebrovascular diseases, other types of cancer, and those patients lost to follow-up. We finally assembled a cohort of 462 PCa patients who had liver function and coagulation measured within 2 weeks before prostate biopsy.

\section{Clinical and pathological evaluation}

Medical data on clinical characteristics including age, prostate-specific antigen (PSA), serum albumin levels and plasma fibrinogen levels at diagnosis, clinical tumor stage, biopsy Gleason score and follow-up information were collected. The pathologic slides were re-reviewed by the urologic pathologists, and the Gleason scores were obtained from the original pathology reports. For clinical tumor stage, patients underwent pelvic Computed Tomography or Magnetic Resonance Imaging. Radionuclide bone scan was performed to determine whether there was bone metastasis. PCa patients were stratified into low-, intermediate-, and high-risk groups according to the EAU guidelines [15].

Eligible patients were treated with continuous ADT as first-line therapy, including castration and antiandrogen therapy. Castration involved surgical or medical castration by using a luteinizing hormone releasing hormone (LHRH) agonist, such as goserelin $3.6 \mathrm{mg}$, administered subcutaneously every month. Antiandrogen therapy was by bicalutamide tablets 50 $\mathrm{mg}$ per day orally or flutamide 3 times a day, $250 \mathrm{mg}$ each time orally.

\section{Follow-up}

Patients were followed for survival information every 3 months. Duration of the follow-up was assessed from the date of treatment until the last follow-up (Mar 2016) or death, which was defined as cancer-related or a different cause. Progression was defined as castration-resistance or death, and the castration-resistance was judged according to the EAU guidelines [16]. The median follow-up duration was 43.69 months (IQR, 30.06-59.60).

\section{Laboratory assays}

Venous blood samples were collected before the prostate biopsy. Serum albumin levels and plasma fibrinogen levels were routinely measured before meals. Plasma fibrinogen levels were measured by the Clauss standard method with bovine thrombin, and serum albumin levels were estimated by bromocresol green albumin method.

\section{Definition of albumin and fibrinogen combined prognostic grade (AFPG)}

The AFPG was calculated according to pretreatment albumin and fibrinogen levels dichotomized by optimal cut-off values and clinical 
reference values. According to receiver operating characteristic (ROC) curve for cancer-specific survival (CSS), the optimal cut-off value of serum albumin levels was $39.55 \mathrm{~g} \mathrm{~L}^{-1}$, the aera under the ROC curve (AUC) was 0.630 with a sensitivity of $79.1 \%$, a specificity of $45.5 \%$ by the Youden index, and the optimal cut-off value of plasma fibrinogen levels was $3.265 \mathrm{~g} \mathrm{~L}^{-1}$, the AUC was 0.681 with a sensitivity of $63.6 \%$, a specificity of $67.4 \%$. The clinical reference values of serum albumin and plasma fibrinogen levels were $35.00 \mathrm{~g} \mathrm{~L}^{-1}$ and $4.00 \mathrm{~g} \mathrm{~L}^{-1}$, respectively. Patients were assigned AFPG 1, 2, or 3 based on the presence of decreased or elevated albumin and fibrinogen levels, as follows: Patients with decreased albumin levels, but elevated fibrinogen levels were assigned as grade 1, patients with both decreased albumin and fibrinogen levels or elevated albumin and fibrinogen levels were assigned as grade 2, and Patients with elevated albumin levels, but decreased fibrinogen levels were assigned as grade 3 .

\section{Statistical analysis}

The Wilcoxon Signed Rank test was used to interrogate the median with interquartile ranges (IQRs) between AFPG and clinical characteristics, while Chi-squared or Fisher's exact tests were used for categorical variables. The survival distributions, including progression-free survival (PFS), cancer-specific survival (CSS) and overall survival (OS) were estimated by the Kaplan-Meier method and compared by a log-rank test, and subgroup analyses were taken according to Gleason score and incidence of metastasis. PFS was calculated from the date of prostate biopsy to the date of disease progression or the time of the last follow-up. The effect of AFPG on PFS, CSS and OS were examined using cox proportional hazard regression models. All variables including AFPG with a $p$-value $<0.05$ on univariate analyses were entered into multivariate stepwise cox regression analyses. Hazard ratio (HR) and $95 \%$ confidence interval (CI) were computed. To examine whether new parameters (albumin, fibrinogen and AFPG) can provide additional prognostic power when combined with basic clinical variables, we built predictive models and calculated the c-indexes using the $\mathrm{R}$ package "survival" by integrating clinical variables with albumin, fibrinogen or AFPG. For each core set, we randomly extracted $20 \%$ samples as the test set to generate a c-index, the above procedure was repeated 100 times to generate 100 c-indexes, and reported as median with IQRs. All tests were two-sided. Differences were considered to be statistically significant if $p$-value $<0.05$. Statistical analysis was carried out using SPSS, version 19.0.

\section{Results}

\section{Clinical characteristics}

The clinical characteristics of the patients are detailed in Table 1. 462 PCa patients were suitable for analysis. The median age of the patients was 76 years old (IQR, 68-79).

Table 1. Clinical characteristics of prostate cancer patients treated with ADT $(n=462)$

\begin{tabular}{ll}
\hline Parameters & Values \\
\hline $\begin{array}{l}\text { Age (median, interquartile range), } \\
\text { years }\end{array}$ & $76(68-79)$ \\
PSA (median, interquartile range), & $78.90(29.78-157.00)$ \\
$\mu \mathrm{g} / \mathrm{L}$ & \\
Gleason Score (n, \%) & \\
$<7$ & $43(9.31)$ \\
$3+4$ & $66(14.28)$ \\
$4+3$ & $112(24.24)$ \\
8 & $153(33.12)$ \\
$>8$ & $88(19.05)$ \\
Metastasis (n, \%) & \\
No & $258(55.84)$ \\
Yes & $204(44.16)$ \\
Risk Stratification (n, \%) & \\
Low & $3(0.65)$ \\
Intermediate & $44(9.52)$ \\
High & $415(89.83)$ \\
Albumin (median, interquartile & $42.09(39.49-44.91)$ \\
range), g/L & \\
Fibrinogen (median, interquartile & $3.07(2.53-3.70)$ \\
range), g/L & \\
AFPG, according to the optimal \\
cut-off values (n, \%)
\end{tabular}

\section{Association between AFPG and clinical and pathological characteristics}

Patients with decreased albumin levels, but elevated fibrinogen levels were assigned as grade 1, patients with both decreased albumin and fibrinogen levels or elevated albumin and fibrinogen levels were assigned as grade 2, and patients with elevated albumin levels, but decreased fibrinogen levels were assigned as grade 3 . 
Table 2. Clinical characteristics of prostate cancer patients according to AFPG

\begin{tabular}{|c|c|c|c|c|c|c|c|c|}
\hline \multirow[t]{2}{*}{ Parameters } & \multicolumn{4}{|c|}{ AFPG, according to optimal cut-off values } & \multicolumn{4}{|c|}{ AFPG, according to clinical reference values } \\
\hline & Grade 1 & Grade 2 & Grade 3 & $P$-value & Grade 1 & Grade 2 & Grade 3 & $P$-value \\
\hline Age (median, interquartile range), years & $78.5(72-82)$ & $76(68-79)$ & $75(66-79)$ & 0.005 & $76.5(62-79)$ & $76(65-82)$ & $76(68-79)$ & 0.908 \\
\hline PSA (median, interquartile range), $\mu \mathrm{g} / \mathrm{L}$ & $125.5(61.4-261.0)$ & $100.0(31.7-154.5)$ & $53.4(24.3-133.6)$ & $<0.001$ & $121(100-261)$ & $100(57.5-214)$ & $64.4(27.7-140.0)$ & 0.002 \\
\hline Gleason Score $(\leq 7 />7)$ & $17 / 41$ & $75 / 105$ & $129 / 95$ & $<0.001$ & $0 / 6$ & $31 / 61$ & $190 / 174$ & $<0.001$ \\
\hline Metastasis (no/yes) & $21 / 37$ & $83 / 97$ & $154 / 70$ & $<0.001$ & $0 / 6$ & $42 / 50$ & $216 / 148$ & $<0.001$ \\
\hline $\begin{array}{l}\text { Risk Stratification } \\
\text { (low-intermediate/high) }\end{array}$ & $3 / 55$ & $14 / 166$ & $30 / 194$ & 0.025 & $0 / 6$ & $4 / 88$ & $43 / 321$ & 0.077 \\
\hline
\end{tabular}

Abbreviations: PSA: prostate-specific antigen; AFPG: pretreatment albumin and fibrinogen combined prognostic grade.

According to optimal cut-off values, all patients were divided into either low albumin group $(\mathrm{n}=118$, $25.54 \%)$ or high albumin group $(\mathrm{n}=344,74.46 \%)$, either low fibrinogen group ( $\mathrm{n}=284,61.47 \%$ ) or high fibrinogen group $(\mathrm{n}=178,38.53 \%)$, either grade $1(\mathrm{n}=$ $58,12.55 \%)$, grade $2(\mathrm{n}=180,38.96 \%)$ or grade $3(\mathrm{n}=$ $224,48.49 \%$ ) of AFPG, and there were significant differences between grade 1, 2, and 3 of AFPG in terms of age $(p=0.005)$, PSA $(p<0.001)$, Gleason score $(p<0.001)$ and incidence of metastasis $(p<0.001)$ and risk stratification $(p=0.025)$.

According to clinical reference values, the number of patients with decreased and elevated albumin levels was 20 (4.33\%) and 442 (95.67\%), respectively; decreased and elevated fibrinogen levels was $378(81.82 \%)$ and $84(18.18 \%)$, respectively; grade 1 , grade 2 and grade 3 of AFPG was $6(1.30 \%), 92$ $(19.91 \%)$ and $364(78.79 \%)$, respectively, and there were significant differences between grade 1, 2, and 3 of AFPG in terms of PSA ( $p=0.002)$, Gleason score $(p$ $<0.001)$ and incidence of metastasis $(p<0.001)$, however, age $(p=0.908)$ and risk stratification $(p=$ 0.077 ) were similar between grade 1, 2, and 3 of AFPG. (Table 2)

\section{Association between AFPG and prognosis of PCa}

The median follow-up duration was 43.69 months, out of 462 patients with usable follow-up information, 203 (43.94\%) patients experienced disease progression, and 109 (23.59\%) patients died, including $88(19.05 \%)$ patients died of PCa at the end of the last follow-up.

According to optimal cut-off values or clinical reference values, the patients with decreased albumin levels or elevated fibrinogen levels or decreased AFPG had a significantly worse survival than those with elevated albumin levels or decreased fibrinogen levels or elevated AFPG with regard to PFS, CSS and OS (Log-rank test, each $p<0.001$, Figure 1 and Figure 2). As shown in Figure 3 and Figure 4, according to optimal cut-off values, in the subgroup of patients with Gleason score $>7$ or bone metastasis, decreased AFPG predicted worse PFS, CSS and OS (Log-rank test, each $p<0.001$ ), however, in the subgroup of Gleason score $\leq 7$ or non-metastasis, the differences of clinical outcomes were not significant between high AFPG group and low AFPG group (Log-rank test, each $p>0.05$ ) except for OS in the subgroup of non-metastasis (Log-rank test, $p=0.005$ ).

Univariate and multivariate cox regression analyses (stepwise analysis) of the factors influencing PFS, CSS and OS were presented in Table 3 and 4. Univariate analyses demonstrated that Gleason score, incidence of metastasis, albumin levels, fibrinogen levels and AFPG according to optimal cut-off values or clinical reference values were significant predictors for PFS, CSS and OS (each $p<0.05$ ), age was a significant predictor for PFS, not for CSS and OS. In the multivariate analyses, we identified that Gleason score, incidence of metastasis and AFPG were independent prognostic indicators for PFS, CSS and OS (each $p<0.01$ ). According to optimal cut-off values, decreased AFPG was independently associated with poor PFS $(\mathrm{HR}=1.306,95 \% \mathrm{CI}$ $0.954-1.789, p=0.096$, grade2 $/ 3 ; \mathrm{HR}=2.601,95 \% \mathrm{CI}$ 1.758-3.847, $p<0.001$, grade1/3), CSS $(\mathrm{HR}=1.717$, 95\% CI 0.989-2.971, $p=0.055$, grade2/3; HR $=4.014$, $95 \%$ CI 2.204-7.309, $p<0.001$, grade1 $/ 3)$ and OS $(\mathrm{HR}=$ $1.449,95 \%$ CI $0.907-2.314, p=0.120$, grade2/3; HR = 3.406, 95\% CI 2.024-5.729, $p<0.001$, grade1/3), respectively. As for clinical reference values, AFPG was also proved to be an independent prognostic factor for PFS $(\mathrm{HR}=1.930,95 \%$ CI 1.393-2.673, $p<$ 0.001 , grade2 $/ 3 ; \mathrm{HR}=3.061,95 \%$ CI 1.330-7.046, $p=$ 0.009, grade1/3), CSS (HR $=2.223,95 \%$ CI 1.407-3.514, $p=0.001$, grade $2 / 3 ; \mathrm{HR}=4.178,95 \%$ CI 1.640-10.648, $p$ $=0.003$, grade $1 / 3)$ and $\mathrm{OS}(\mathrm{HR}=1.939,95 \% \mathrm{CI}$ $1.276-2.945, p=0.002$, grade2 $/ 3 ; \mathrm{HR}=3.738,95 \% \mathrm{CI}$ 1.480-9.440, $p=0.005$, grade1/3), respectively.

The predictive accuracy was calculated and presented in Table 5. In the univariate analysis, the c-indexes of AFPG according to optimal cut-off values for PFS, CSS and OS were 0.632, 0.704 and 0.677, respectively, which were higher than that of Gleason score, albumin levels, fibrinogen levels or AFPG according to clinical reference values but not incidence of metastasis. In the multivariate analysis, the predictive accuracy of the base model including the traditional predictor variables of Gleason score and incidence of metastasis for PFS, CSS and OS was 
$72.7 \%, 80.0 \%$ and $74.6 \%$, respectively; with the addition of albumin levels according to optimal cut-off values, the predictive accuracy was $74.4 \%$, $83.2 \%$ and $78.3 \%$, respectively; with the addition of albumin levels according to clinical reference values, the predictive accuracy was $73.3 \%, 81.5 \%$ and $75.7 \%$, respectively; with the addition of fibrinogen levels according to optimal cut-off values, the predictive accuracy was $74.5 \%, 82.5 \%$ and $76.6 \%$, respectively; with the addition of fibrinogen level according to clinical reference values, the predictive accuracy was $73.9 \%, 82.2 \%$ and $76.3 \%$, respectively; with the addition of AFPG according to optimal cut-off values, the predictive accuracy was $75.0 \%, 84.0 \%$ and $79.0 \%$, respectively; with the addition of albumin level according to clinical reference values, the predictive accuracy was $74.4 \%, 82.8 \%$ and $76.7 \%$, respectively. our new model integrating Gleason score, incidence of metastasis and AFPG according to optimal cut-off values retained its advantage than the base model, or other integrated models, including the traditional predictors and albumin levels or fibrinogen levels or AFPG according to clinical reference values.

\section{Discussion}

Prostate cancer (PCa) is the most commonly diagnosed cancer and the second leading cause of cancer death in men in the United States [17]. Androgen deprivation therapy (ADT) is the main treatment for aged or advanced or metastatic PCa. Despite recent progress in the identification of genetic and molecular alterations in $\mathrm{PCa}$, the routine prognostic risk assessment of PCa patients currently relies on traditional clinicopathological prognostic factors, including Gleason score, clinical tumor stage, serum PSA level, and metastasis at the time of diagnosis. The predictive accuracy of this traditional prognostic model needs be further improved by the incorporation of novel prognostic biomarkers.
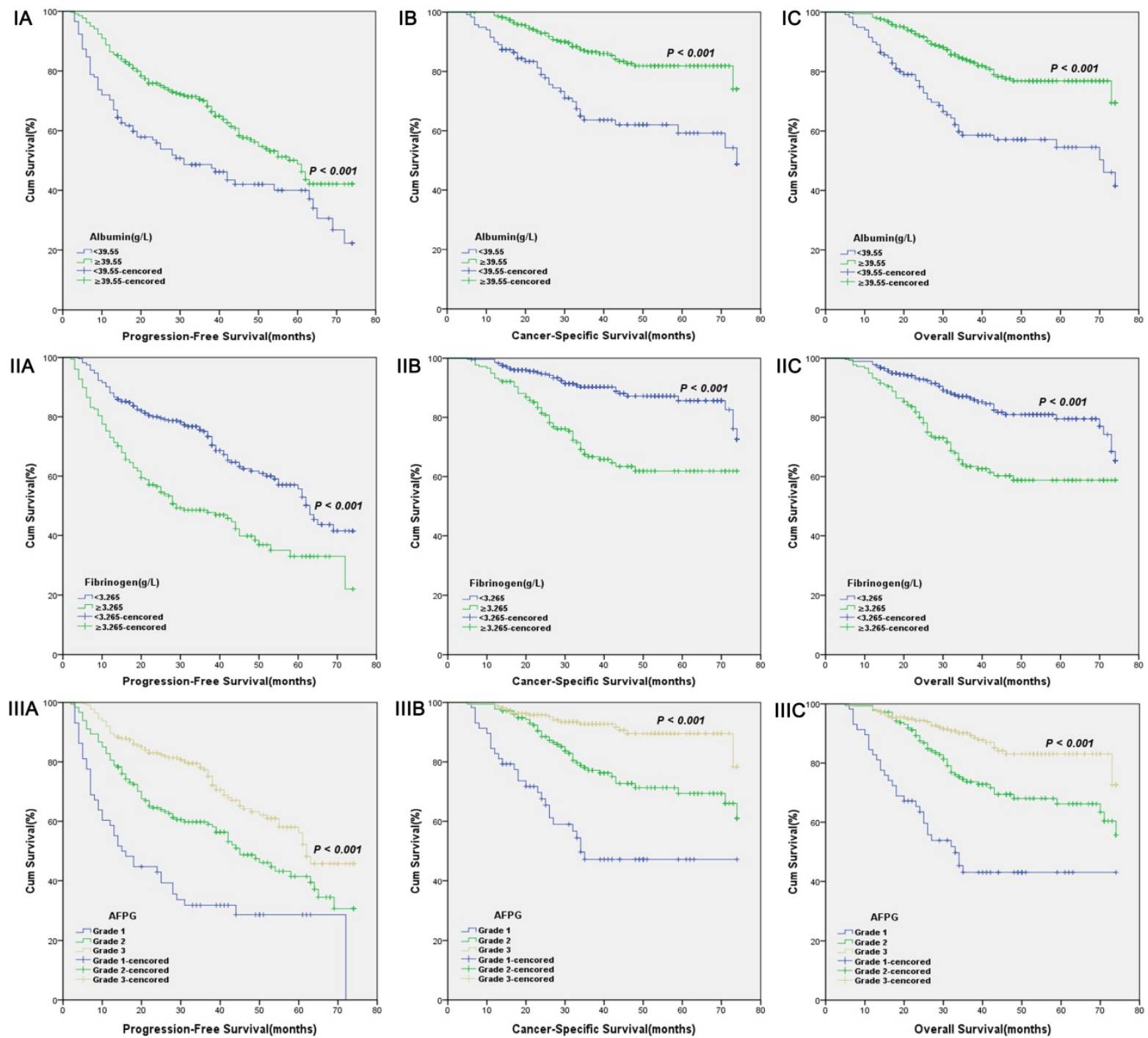

Figure 1. Kaplan-Meier curves for albumin levels (I), fibrinogen levels (II) or AFPG according to the optimal cut-off values (III). A. Progression-free survival (PFS), B. Cancer-specific survival (CSS) and C. Overall survival (OS). 

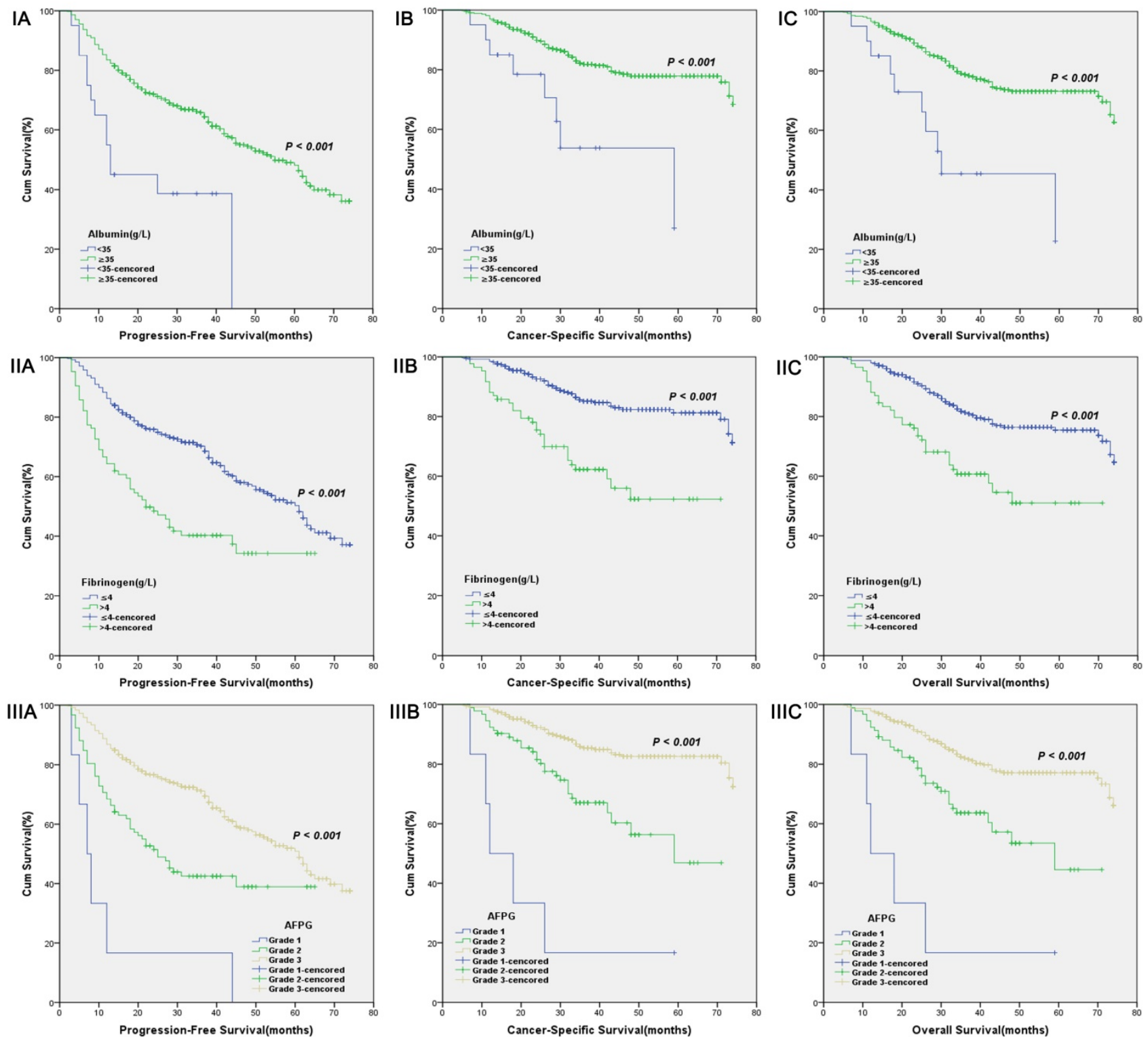

Figure 2. Kaplan-Meier curves for albumin levels (I), fibrinogen levels (II) or AFPG according to the clinical reference values (III). A. Progression-free survival (PFS), B. Cancer-specific survival (CSS) and C. Overall survival (OS).

The serum albumin levels and plasma fibrinogen levels are routinely measured blood-based parameters. In this large cohort of $\mathrm{PCa}$ patients treated with $\mathrm{ADT}$, we found that pretreatment decreased albumin and fibrinogen combined prognostic grade (AFPG) was associated with increased risk for disease recurrence, cancer specific mortality and all-cause mortality. These findings remained significant after adjusting for clinicopathological features, indicating an independent association of pretreatment decreased AFPG with adverse outcomes.

To the best of our knowledge, our analysis is the first study to take albumin and fibrinogen together to evaluate whether the combination of them could present a better predictive value for PCa patients' clinical outcome, and strikingly we got satisfactory results. Our study showed that AFPG was an independent prognostic indicator for PFS, CSS and OS. Additionally, we made a subgroup analysis and built a new integrated prognostic model. In subgroup analysis, we found that decreased AFPG according to optimal cut-off values could predict poor prognosis in the subgroup of patients with Gleason score $>7$ or bone metastasis. In the subgroup of Gleason score $\leq 7$ or non-metastasis, however, AFPG was not statistically significantly associated with prognosis except for OS in the subgroup of non-metastasis, probably because the percentages of patients who reached the endpoints (progression, cancer-related death and overall death) in these subgroups were too small. 

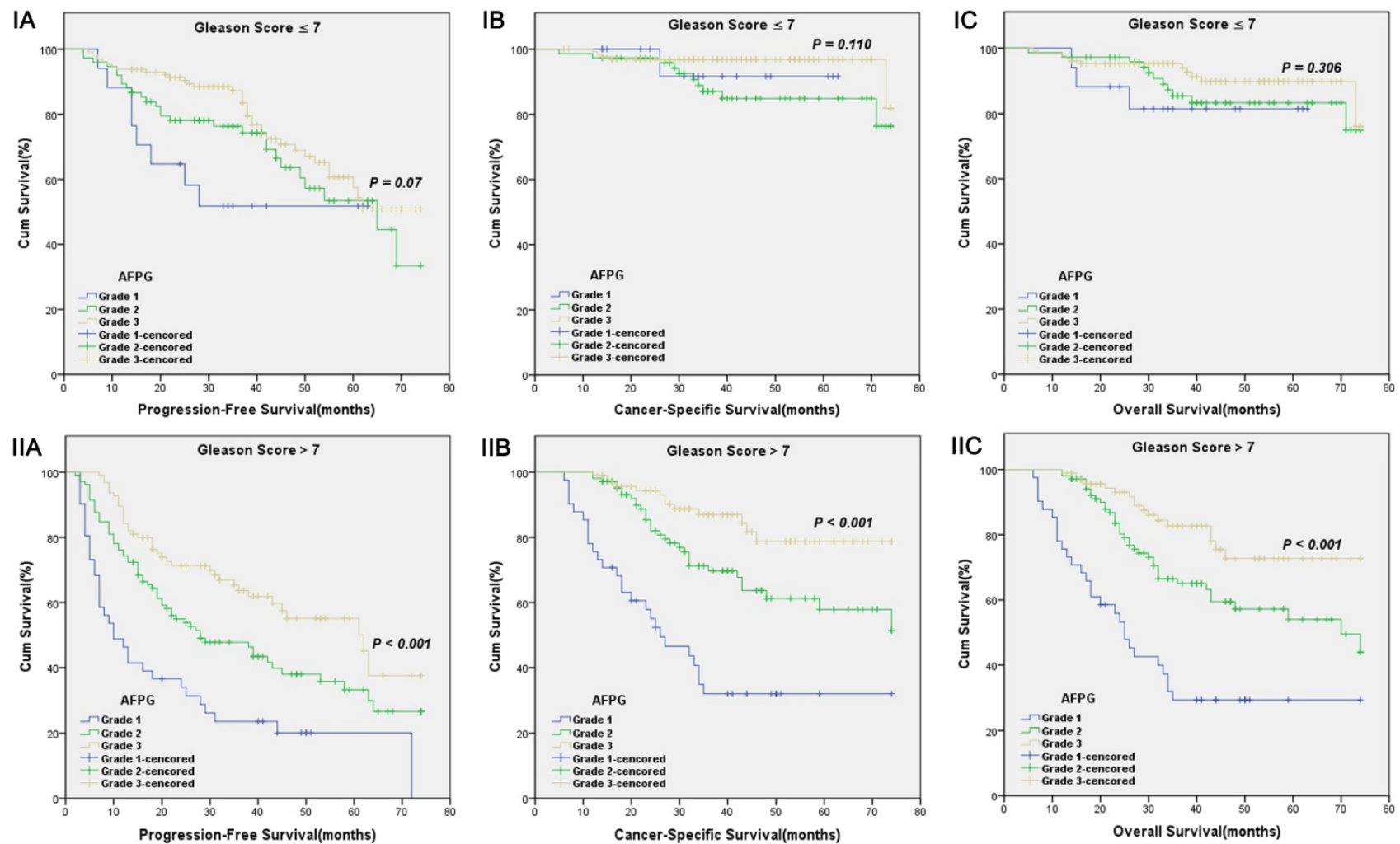

Figure 3. Kaplan-Meier survival curves stratified by AFPG according to the optimal cut-off values in prostate cancer patients with Gleason score $\leq 7(\mathrm{I})$ and $\mathrm{Gleason}$ score > 7(II). A. Progression-free survival (PFS), B. Cancer-specific survival (CSS) and C. Overall survival (OS).
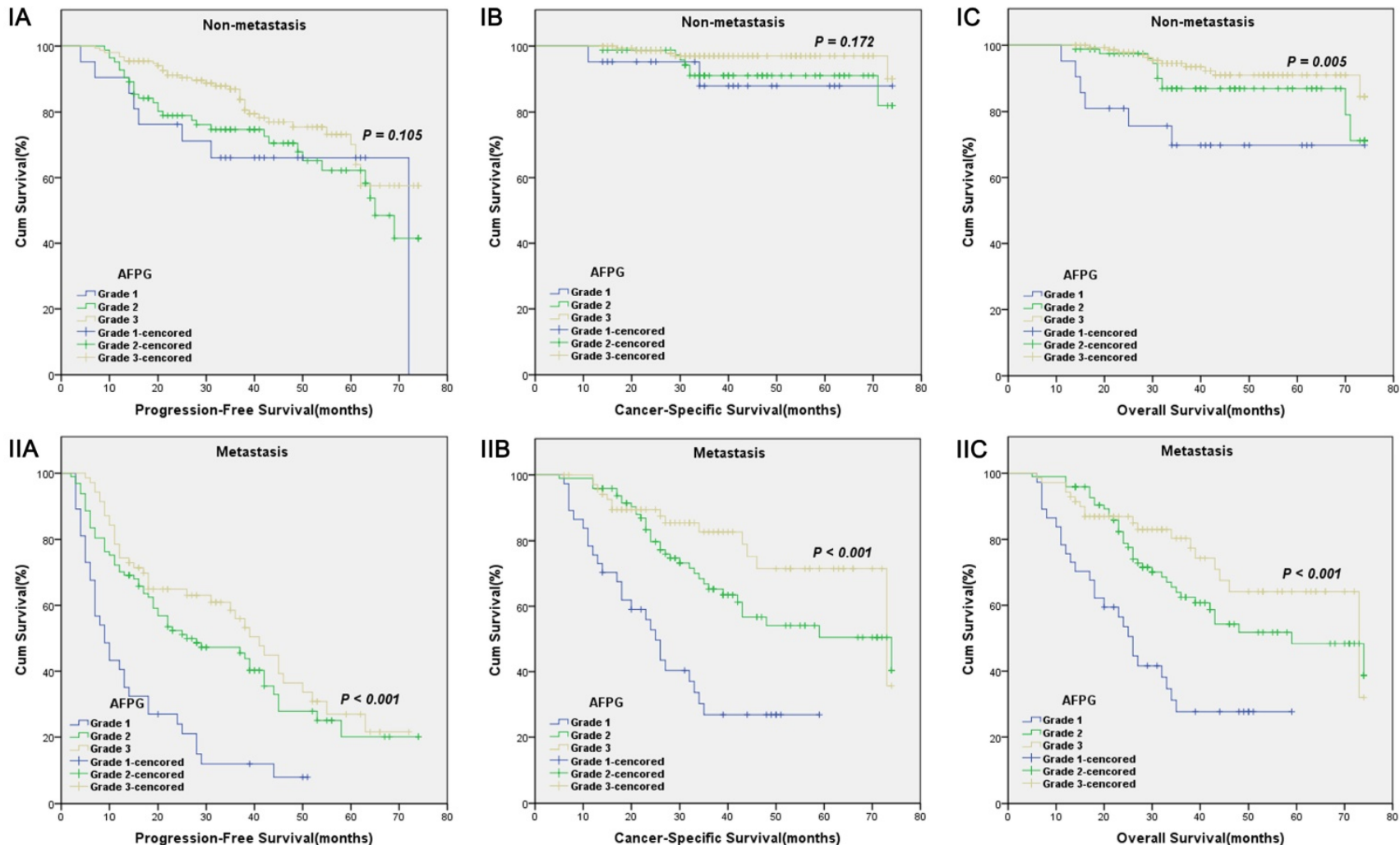

Figure 4. Kaplan-Meier survival curves stratified by AFPG according to the optimal cut-off values in prostate cancer patients with non-metastasis(I) and metastasis(II). A. Progression-free survival (PFS), B. Cancer-specific survival (CSS) and C. Overall survival (OS). 
Table 3. Univariate analyses of various clinical parameters in prostate cancer patients

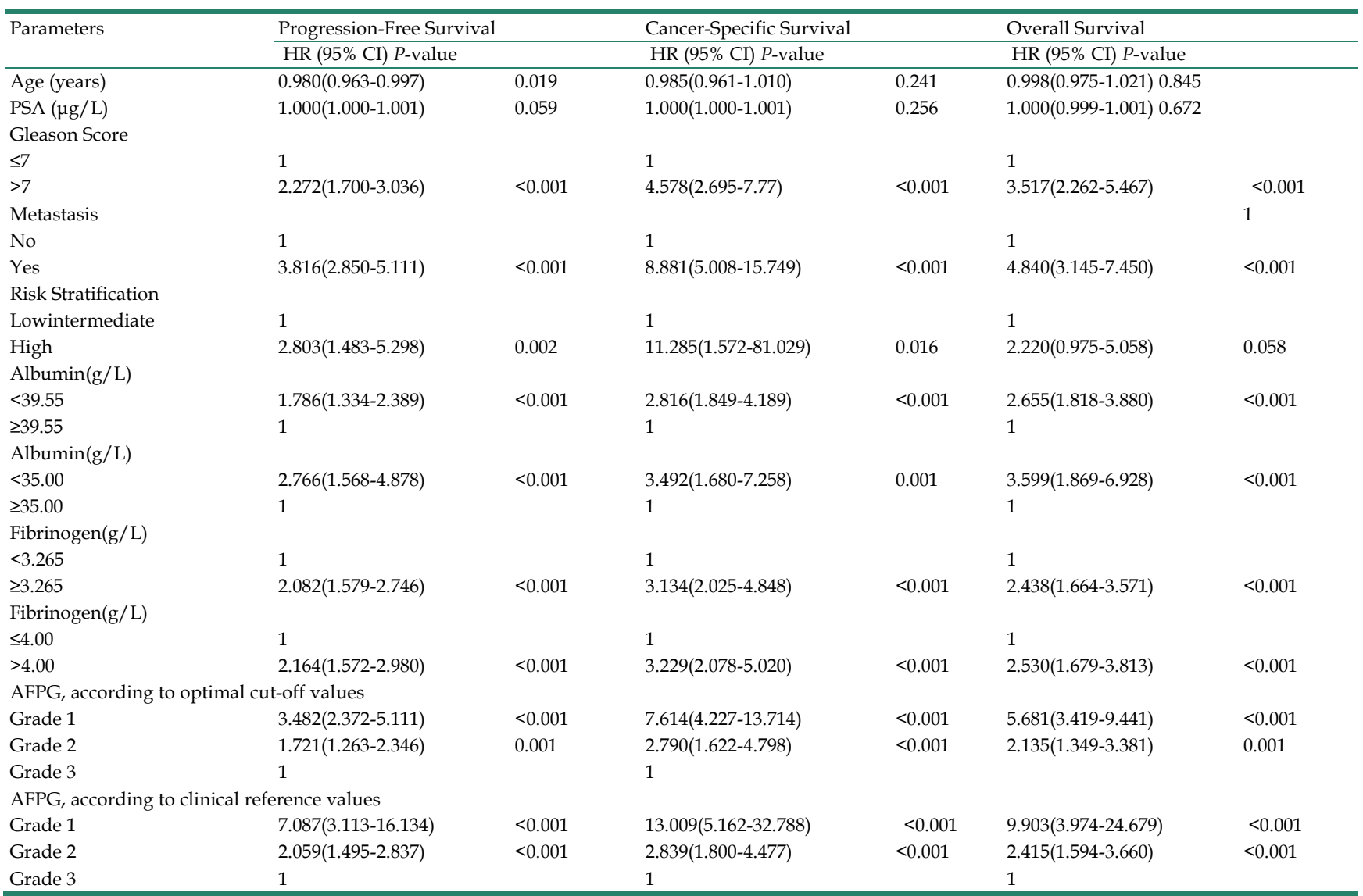

Abbreviations: HR: hazard ratio; CI: confidence interval; PSA: prostate-specific antigen; AFPG: pretreatment albumin and fibrinogen combined prognostic grade.

Table 4. Multivariate analyses of various clinical parameters in prostate cancer patients

\begin{tabular}{|c|c|c|c|c|c|c|c|c|c|c|c|c|}
\hline \multirow[t]{3}{*}{ Parameters } & \multicolumn{6}{|c|}{ According to optimal cut-off values } & \multicolumn{6}{|c|}{ According to clinical reference values } \\
\hline & \multicolumn{2}{|c|}{ Progression-Free Survival } & \multicolumn{2}{|c|}{ Cancer-Specific Survival } & \multicolumn{2}{|c|}{ Overall Survival } & \multicolumn{2}{|c|}{ Progression-Free Survival } & \multicolumn{2}{|c|}{ Cancer-Specific Survival } & \multicolumn{2}{|c|}{ Overall Survival } \\
\hline & $\mathrm{HR}(95 \% \mathrm{CI})$ & $P$-value & HR $(95 \% \mathrm{CI})$ & $P$-value & HR $(95 \% \mathrm{CI})$ & $P$-value & HR $(95 \% \mathrm{CI})$ & $P$-value & HR $(95 \% \mathrm{CI})$ & $P$-value & HR $(95 \%$ CI) & $P$-value \\
\hline Age (years) & & 0.096 & - & - & - & - & - & 0.440 & - & - & - & - \\
\hline \multicolumn{13}{|c|}{ Gleason Score } \\
\hline$\leq 7$ & 1 & & 1 & & 1 & & 1 & & 1 & & 1 & \\
\hline$>7$ & $\begin{array}{l}1.703(1.265-2 . \\
293)\end{array}$ & $<0.001$ & $\begin{array}{l}2.890(1.687-4.95 \\
1)\end{array}$ & $<0.001$ & $\begin{array}{l}2.468(1.574-3 . \\
870)\end{array}$ & $<0.001$ & $\begin{array}{l}1.699(1.261-2 . \\
288)\end{array}$ & $<0.001$ & $\begin{array}{l}2.888(1.682-4.95 \\
9)\end{array}$ & $<0.001$ & $\begin{array}{l}2.464(1.568-3 . \\
872)\end{array}$ & $<0.001$ \\
\hline \multicolumn{13}{|l|}{ Metastasis } \\
\hline No & 1 & & 1 & & 1 & & 1 & & 1 & & 1 & \\
\hline Yes & $\begin{array}{l}3.236(2.398-4 \\
368)\end{array}$ & $<0.001$ & $\begin{array}{l}6.156(3.433-11.0 \\
39)\end{array}$ & $<0.001$ & $\begin{array}{l}3.546(2.276-5 \\
526)\end{array}$ & $<0.001$ & $\begin{array}{l}3.420(2.538-4 . \\
608)\end{array}$ & $<0.001$ & $\begin{array}{l}6.916(3.873-12.3 \\
51)\end{array}$ & $<0.001$ & $\begin{array}{l}3.893(2.510-6 \\
039)\end{array}$ & $<0.001$ \\
\hline \multicolumn{13}{|l|}{ AFPG } \\
\hline Grade 1 & $\begin{array}{l}\text { 2.601(1.758-3. } \\
847)\end{array}$ & $<0.001$ & $\begin{array}{l}\text { 4.014(2.204-7.30 } \\
\text { 9) }\end{array}$ & $<0.001$ & $\begin{array}{l}3.406(2.024-5 . \\
729)\end{array}$ & $<0.001$ & $\begin{array}{l}3.061(1.330-7 \\
046)\end{array}$ & 0.009 & $\begin{array}{l}4.178(1.640-10.6 \\
48)\end{array}$ & 0.003 & $\begin{array}{l}3.738(1.480-9 . \\
440)\end{array}$ & 0.005 \\
\hline Grade 2 & $\begin{array}{l}1.306(0.954-1 . \\
789)\end{array}$ & 0.096 & $\begin{array}{l}1.717(0.989-2.97 \\
1)\end{array}$ & 0.055 & $\begin{array}{l}1.449(0.907-2 \\
314)\end{array}$ & 0.120 & $\begin{array}{l}1.930(1.393-2 . \\
673)\end{array}$ & $<0.001$ & $\begin{array}{l}2.223(1.407-3.51 \\
4)\end{array}$ & 0.001 & $\begin{array}{l}1.939(1.276-2 . \\
945)\end{array}$ & 0.002 \\
\hline Grade 3 & 1 & & 1 & & 1 & & 1 & & 1 & & 1 & \\
\hline
\end{tabular}

Abbreviations: HR: hazard ratio; CI: confidence interval; PSA: prostate-specific antigen; AFPG: pretreatment albumin and fibrinogen combined prognostic grade.

In our cohort, the predictive accuracy of albumin levels or fibrinogen levels or AFPG according to optimal cut-off values was higher than that of albumin levels or fibrinogen levels or AFPG according to clinical reference values. This might be due to small changes of nutritional and inflammatory parameters for patients initially diagnosed as $\mathrm{PCa}$, and they were still in normal clinical reference ranges. Moreover, the sample size of decreased albumin levels, elevated fibrinogen levels or grade 1 (AFPG) according to clinical reference values was too tiny. Therefore, the optimal cut-off values may be more suitable for dichotomy of these patients than the clinical reference values. In the multivariate model analyses, our new model integrating Gleason score, incidence of metastasis and AFPG according to optimal cut-off values showed great advantage than the base model, including Gleason score and incidence of metastasis, 
or other integrated models, including Gleason score, incidence of metastasis and albumin levels or fibrinogen levels or AFPG according to clinical reference values, indicating AFPG according to optimal cut-off values was a strong independent prognostic predictor for PCa and it based integrated model had a higher predictive accuracy than the base or other integrated models.

In this present study, patients with decreased AFPG were more likely to have higher PSA, Gleason score and incidence of metastasis. In our multivariate cox analyses, Gleason score and incidence of metastasis were independent predictors. Taken together, these differences of tumor characteristics may partly explain why the patients with decreased AFPG in our cohort had more aggressive disease.

The mechanisms responsible for this observation remains unclear and is yet to be elucidated. However, several previous experimental and clinical studies supported the observation of our study. A decreased AFPG includes both decreased albumin level and elevated fibrinogen level, which may be both associated with cancer progression and poor outcome. Gupta D et al. [10] performed a meta-analysis and found an association of higher serum albumin level with better survival of various tumors. Decreased albumin level not only reflcets malnutrition status, but also indicates existence of inflammation in the host. Malnutrition is highly prevalent in cancer patients, and may produce a great deal of negative consequences, such as impaired immune functions, reduced response to cancer treatment and shortened overall survival [18]. As part of systemic inflammatory response to the tumor or from the tumor itself, inflammatory mediators including interleukin-1 (IL-1), IL-6, necrosis factor a and acute phase reactants were released, which might increase the transcapillary escape rate of albumin and modulate albumin synthesis by hepatocytes [19-22], thus albumin level could serve as a good indicator of prognosis for cancer. As for fibrinogen level, numerous studies have released its role in the development of cancer, metastatic spread and prognostic assessment [14, 23-25]. Yano HJ et al. [23] showed that fibrinogen interacted with multiple integrin and non-integrin receptors of cancer cells to regulate tumor cell proliferation, migration, and signaling. Sahni A et al. [24] noted that fibrinogen may bind to vascular endothelial cell growth factors, and stimulate endothelial cell proliferation and angiogenesis. Ziaran et al. [25] demonstrated a significant increase of fibrinogen levels in prostate cancer patients after 12 months of androgen deprivation therapy, indicating fibrinogen may be association with tolerance of ADT. Our previous study showed an association between elevated plasma fibrinogen with poor clinical outcome for $\mathrm{PCa}$ patients [14]. Besides, as an acute-phase reaction protein [10], fibrinogen level would be increased during systemic response of host to tumor.

There are some limitations to our current study. First, this was a retrospective investigation. Despite the strict enrollment criteria applied, we were unable to completely exclude other conditions that might cause hematologic and nutritional changes in PCa. Second, $\mathrm{T}$ and $\mathrm{N}$ stage were not showed for that $15 \%$ PCa patients with serum PSA level $>20 \mu \mathrm{g} / \mathrm{L}$ refused CT or MRI scan to evaluate tumor invasion depth and determine whether there was lymph node metastasis. Our results need to be validated by prospective research and patient data from other parts of the world.

Table 5. Univarate and multivariate models of possible independent prognostic variables in prostate cancer patients.

\begin{tabular}{|c|c|c|c|c|c|c|}
\hline \multirow[t]{2}{*}{ Parameters } & \multicolumn{2}{|c|}{ Progression-Free Survival } & \multicolumn{2}{|c|}{ Cancer-Specific Survival } & \multicolumn{2}{|c|}{ Overall Survival } \\
\hline & $\begin{array}{l}\text { Univarate } \\
\text { Median(IQR) }\end{array}$ & $\begin{array}{l}\text { Multivarate } \\
\text { Median(IQR) }\end{array}$ & $\begin{array}{l}\text { Univarate } \\
\text { Median(IQR) }\end{array}$ & $\begin{array}{l}\text { Multivarate } \\
\text { Median(IQR) }\end{array}$ & $\begin{array}{l}\text { Univarate } \\
\text { Median(IQR) }\end{array}$ & $\begin{array}{l}\text { Multivarate } \\
\text { Median(IQR) }\end{array}$ \\
\hline $\begin{array}{l}\text { Gleason Score } \\
(\leq 7 />7)\end{array}$ & $\begin{array}{l}0.619 \\
(0.598-0.650)\end{array}$ & 0.727 & $\begin{array}{l}0.676 \\
(0.631-0.710)\end{array}$ & 0.800 & $\begin{array}{l}0.646 \\
(0.609-0.682)\end{array}$ & 0.746 \\
\hline $\begin{array}{l}\text { Metastasis } \\
\text { (no/yes) }\end{array}$ & $\begin{array}{l}0.680 \\
(0.658-0.702)\end{array}$ & $(0.703-0.753)$ & $\begin{array}{l}0.744 \\
(0.712-0.772)\end{array}$ & $(0.768-0.837)$ & $\begin{array}{l}0.690 \\
(0.668-0.724)\end{array}$ & $(0.720-0.781)$ \\
\hline $\begin{array}{l}\operatorname{Albumin}(\mathrm{g} / \mathrm{L}) \\
(<39.55 / \geq 39.55)\end{array}$ & $\begin{array}{l}0.580 \\
(0.555-0.601)\end{array}$ & $\begin{array}{l}0.744 \\
(0.719-0.766)\end{array}$ & $\begin{array}{l}0.625 \\
(0.596-0.664)\end{array}$ & $\begin{array}{l}0.832 \\
(0.796-0.857)\end{array}$ & $\begin{array}{l}0.620 \\
(0.592-0.658)\end{array}$ & $\begin{array}{l}0.783 \\
(0.749-0.810)\end{array}$ \\
\hline $\begin{array}{l}\operatorname{Albumin}(\mathrm{g} / \mathrm{L}) \\
(<35 / \geq 35)\end{array}$ & $\begin{array}{l}0.525 \\
(0.512-0.540)\end{array}$ & $\begin{array}{l}0.733 \\
(0.709-0.761)\end{array}$ & $\begin{array}{l}0.529 \\
(0.511-0.557)\end{array}$ & $\begin{array}{l}0.815 \\
(0.782-0.845)\end{array}$ & $\begin{array}{l}0.528 \\
(0.514-0.552)\end{array}$ & $\begin{array}{l}0.757 \\
(0.733-0.797)\end{array}$ \\
\hline $\begin{array}{l}\text { Fibrinogen }(\mathrm{g} / \mathrm{L}) \\
(<3.225 / \geq 3.225)\end{array}$ & $\begin{array}{l}0.606 \\
(0.585-0.637)\end{array}$ & $\begin{array}{l}0.745 \\
(0.712-0.767)\end{array}$ & $\begin{array}{l}0.652 \\
(0.616-0.697)\end{array}$ & $\begin{array}{l}0.825 \\
(0.785-0.857)\end{array}$ & $\begin{array}{l}0.622 \\
(0.595-0.665)\end{array}$ & $\begin{array}{l}0.766 \\
(0.737-0.800)\end{array}$ \\
\hline $\begin{array}{l}\text { Fibrinogen }(\mathrm{g} / \mathrm{L}) \\
(\leq 4 />4)\end{array}$ & $\begin{array}{l}0.578 \\
(0.560-0.601)\end{array}$ & $\begin{array}{l}0.739 \\
(0.711-0.769)\end{array}$ & $\begin{array}{l}0.615 \\
(0.591-0.659)\end{array}$ & $\begin{array}{l}0.822 \\
(0.785-0.859)\end{array}$ & $\begin{array}{l}0.595 \\
(0.562-0.623)\end{array}$ & $\begin{array}{l}0.763 \\
(0.739-0.799)\end{array}$ \\
\hline $\begin{array}{l}\text { AFPG, according to the } \\
\text { optimal cut-off values }\end{array}$ & $\begin{array}{l}0.632 \\
(0.615-0.667)\end{array}$ & $\begin{array}{l}0.750 \\
(0.722-0.774)\end{array}$ & $\begin{array}{l}0.704 \\
(0.657-0.744)\end{array}$ & $\begin{array}{l}0.840 \\
(0.800-0.875)\end{array}$ & $\begin{array}{l}0.677 \\
(0.639-0.723)\end{array}$ & $\begin{array}{l}0.790 \\
(0.747-0.819)\end{array}$ \\
\hline $\begin{array}{l}\text { AFPG, according to } \\
\text { clinical reference values }\end{array}$ & $\begin{array}{l}0.589 \\
(0.568-0.612)\end{array}$ & $\begin{array}{l}0.744 \\
(0.716-0.771)\end{array}$ & $\begin{array}{l}0.619 \\
(0.592-0.662)\end{array}$ & $\begin{array}{l}0.828 \\
(0.789-0.862)\end{array}$ & $\begin{array}{l}0.604 \\
(0.578-0.637)\end{array}$ & $\begin{array}{l}0.767 \\
(0.738-0.807)\end{array}$ \\
\hline
\end{tabular}

Abbreviations: IQR: interquartile range; AFPG: pretreatment albumin and fibrinogen combined prognostic grade; Multivariate models include Gleason score and metastasis. 
In conclusion, our study firstly proposed a new index named AFPG to predict clinical outcomes of PCa receiving ADT by taking pretreatment serum albumin levels and plasma fibrinogen levels together. We not only demonstrated that decreased AFPG could predict a significantly poor prognosis in patients with PCa, but also found AFPG according to optimal cut-off values showed great advantage than albumin levels alone or fibrinogen levels alone or AFPG according to clinical reference values in predicting prognosis. Thus, we recommend adding AFPG according to optimal cut-off values to traditional prognostic model to improve the predictive accuracy.

\section{Acknowledgements}

This study was supported by National Natural Science Foundation of China (81572536, 81672850), Science and Technology Commission of Shanghai Municipality (14140901700, 16411969800), the Joint Research Foundation for Innovative Medical Technology of Shanghai Shenkang Hospital Development Center (SHDC12015125), Shanghai Municipal Education Commission (15ZZ058), Shanghai Municipal Commission of Health and Family Planning (201640247), Shanghai Municipal Education Commission-Gaofeng Clinical Medicine Grant Support (20152215), Key Disciplines Group Construction Project of Pudong Health Bureau of Shanghai (PWZxq2014-05), the Shanghai Jiao Tong University School of Medicine Translational Research Innovation Fund (15ZH4002), and Incubating Program for clinical Research and Innovation of Renji Hospital Shanghai Jiao Tong University School of Medicine (PYZY 16-008, PYXJS16-015).

\section{Competing Interests}

The authors have declared that no competing interest exists.

\section{References}

1. Chen W, Zheng R, Baade PD, et al. Cancer statistics in China, 2015. CA Cancer J Clin. 2016; 66: 115-132.

2. Nelson JB. Hormone therapy for prostate cancer. In: Kavoussi LR, Partin AW, Novick AC, ed. Campbell-Wash Urology,10th ed. Amsterdam: Elsevier; 2012: 2934-2953.

3. Sánchez-Lara K, Turcott JG, Juárez E, et al. Association of nutrition parameters including bioelectrical impedance and systemic inflammatory response with quality of life and prognosis in patients with advanced non-small-cell lung cancer: a prospective study. Nutr Cancer. 2012; 64: 526-534.

4. Gu W, Zhang G, Sun L, et al. Nutritional screening is strongly associated with overall survival in patients treated with targeted agents for metastatic renal cell carcinoma. J Cachexia Sarcopenia Muscle. 2015; 6: 222-230.

5. Saito K, Kihara K. Role of C-reactive protein in urological cancers: a useful biomarker for predicting outcomes. Int J Urol. 2013; 20: 161-171.

6. Fiala $\mathrm{O}$, Pesek M, Finek J, et al. Serum albumin is a strong predictor of survival in patients with advanced-stage non-small cell lung cancer treated with erlotinib. Neoplasma. 2016; 63: 471-476.

7. Sejima $\mathrm{T}$, Iwamoto $\mathrm{H}$, Masago $\mathrm{T}$, et al. Low pre-operative levels of serum albumin predict lymph node metastases and ultimately correlate with a biochemical recurrence of prostate cancer in radical prostatectomy patients. Cent European J Urol. 2013; 66: 126-132.

8. Langsenlehner T, Thurner EM, Krenn-Pilko S, et al. Validation of the neutrophil-to-lymphocyte ratio as a prognostic factor in a cohort of European prostate cancer patients. World J Urol. 2015; 33: 1661-1667.

9. Langsenlehner T, Pichler M, Thurner EM, et al. Evaluation of the platelet-to-lymphocyte ratio as a prognostic indicator in a European cohort of patients with prostate cancer treated with radiotherapy. Urol Oncol. 2015; 33: 201.e9-16.

10. Gupta D, Lis CG. Pretreatment serum albumin as a predictor of cancer survival: a systematic review of the epidemiological literature. Nutr J. 2010; 9; 69.

11. Shafique K, Proctor MJ, McMillan DC, et al. The modified Glasgow prognostic score in prostate cancer: results from a retrospective clinical series of 744 patients. BMC Cancer. 2013; 13: 292.

12. Sun $\mathrm{H}, \mathrm{Hu} \mathrm{P}$, Shen $\mathrm{H}$, et al. Albumin and Neutrophil Combined Prognostic Grade as a New Prognostic Factor in Non-Small Cell Lung Cancer: Results from a Large Consecutive Cohort. PLoS One. 2015; 10: e0144663.

13. Pastore CA, Orlandi SP, Gonzalez MC. The Inflammatory-Nutritional Index; assessing nutritional status and prognosis in gastrointestinal and lung cancer patients. Nutr Hosp. 2014; 29(3): 629-634.

14. Wang Y, Yin W, Wang Z, et al. Pretreatment plasma fibrinogen as an independent prognostic indicator of prostate cancer patients treated with androgen deprivation therapy. Prostate Cancer and Prostatic Diseases. 2016; 19: 209-215.

15. Heidenreich A, Bastian PJ, Bellmunt J, et al. EAU guidelines on prostate cancer. part 1: screening, diagnosis, and local treatment with curative intent-update 2013. Eur Urol. 2014; 65: 124-137.

16. Heidenreich A, Bastian PJ, Bellmunt J, et al. EAU guidelines on prostate cancer. part II: Treatment of advanced, relapsing, and castration-resistant prostatecancer. Eur Urol. 2014; 65: 467-479.

17. Siegel R, Miller KD, Jemal A. Cancer statistics, 2016. CA Cancer J Clin. 2016; 66:7-30.

18. Van Cutsem E, Arends J. The causes and consequences of cancer-associated malnutrition. Eur J Oncol Nurs. 2005; 9 (Suppl 2): S51-S63.

19. Fleck A, Raines G, Hawker F, et al. Increased vascular permeability: a major cause of hypoalbuminaemia in disease and injury. Lancet. 1985; 1: 781-784.

20. Deehan DJ, Heys SD, Simpson W, et al. Correlation of serum cytokine and acute phase reactant levels with alterations in weight and serum albumin in patients receiving immunotherapy with recombinant IL-2. Clin Exp Immunol. 1994; 95: 366-372.

21. Nakashima J, Tachibana $M$, Ueno $M$, et al. Association between tumor necrosis factor in serum and cachexia in patients with prostate cancer. Clin Cancer Res. 1998; 4: 1743-1748.

22. Mantovani A, Allavena P, Sica A, et al. Cancer-related inflammation. Nature. 2008; 454: 436-444.

23. Yano HJ, Hatano K, Tsuno N, et al. Clustered cancer cells show a distinct adhesion behavior from single cell form under physiological shear conditions. J Exp Clin Cancer Res. 2001; 20: 407-412.

24 Sahni A, Francis CW. Vascular endothelial growth factor binds to fibrinogen and fibrin and stimulates endothelial cell proliferation. Blood. 2000; 96: 3772-3778.

25. Ziaran S, Goncalves FM, Breza J Sr. Patients with prostate cancer treated by ADT have significantly higher fibrinogenemia than healthy control. World J Urol. 2013; 31: 289-292. 